\title{
La pedagogía y la motricidad humana, una condición de posibilidad para la construcción de una episteme de la educación física en la perspectiva del desarrollo humano en Colombia
}

Pedagogy and the human motor function: a necessary condition for building a physical education episteme from a Colombian human development perspective

\author{
Jesús María Pinillos García*
}

\section{Resumen}

Palabras clave: Historia de la Educación Física en Colombia, pedagogía del Cuerpo, pedagogía de la Motricidad, expresiones motrices, ocio,

competencia motriz.

\section{Abstract}

Key words:

El artículo se apoya en los resultados de investigaciones de tipo histórico, en los que se indaga por el proceso de constitución de la Educación Física en Colombia, como un campo de saber y de poder. En éste se pretende hacer visible una episteme de la Educación Física contemporánea, fundamentada en la apropiación de los discursos de la Pedagogía y la Motricidad en Colombia por parte de ésta. El análisis muestra inicialmente un campo de saber en medio de una crisis "epistemológica" y "profesional", debido a la diversificación de sus prácticas, situación que se ha presentado en otros campos de saber, el ejemplo más cercano se puede encontrar en la Pedagogía, su referente principal; es más, es muy probable que la tan mencionada "crisis" de la Educación Física sea un legado de la crisis de la Pedagogía.

Posteriormente se hace visible la emergencia a partir de los resultados de investigaciones en el campo de la Educación Física de conceptos como Pedagogía del Cuerpo, Pedagogía de la Motricidad, Expresiones Motrices y Ocio. Los cuales muestran niveles de filiación o articulación y condiciones de posibilidad para la construcción de una episteme, desde una tendencia que orienta sus búsquedas en la perspectiva del desarrollo humano, utilizando como medio la Motricidad humana y el Ocio, entendidos como necesidades humanas.

The present article is supported by the results of historical researches, focused on the building of Physical Education as a field of knowledge and power. The study attempts to bring to light an episteme of contemporary Physical education. An episteme which is grounded on its appropriation of the pedagogy and motor function discourses in Colombia. At first, the analysis shows a field of knowledge in the middle of an "epistemological" and "professional" crisis, due to the diversification of its practices. This situation is not new in other fields of knowledge, with the closest example being Pedagogy, its main benchmark. Furthermore, the so-called Physical education "crisis" is very likely a legacy of the Pedagogy crisis.

Later, the study shows the emergence of concepts such as "body pedagogy", "motor function pedagogy", "motor function expressions" and "leisure", all of them derived from the results of researches in Physical education. These concepts show some level of articulation and probable conditions for the construction of an episteme, from a human development perspective-targeted tendency, by means of the consideration of human motor function and leisure as human needs.

The history of Physical education in Colombia, body pedagogy, motor function pedagogy, motor function expressions, leisure, motor competence.

Fecha de recepción: 5 de octubre de 2007.

Fecha de aceptación: 2 de noviembre de 2007.

\footnotetext{
* Profesor investigador Departamento de Educación Física, Recreación y Deporte de la Facultad de Ciencias Naturales, Exactas y de la Educación de la Universidad del Cauca. Miembro del grupo de Investigación Epistemología e Historia de de la Educación Física, la Recreación y el Deporte.jpinillo@msn.com.jpinillos@unicauca.edu.co

1 El artículo hace parte de la ponencia presentada en el III Encuentro Internacional de Experiencias significativas en Motricidad humana y Educación Física, Recreación y Deporte. Popayán. (Colombia). 6, 7 y 8 de septiembre de de 2006, así como en el Coloquio Regional de Investigación en Educación y Pedagogía en la ciudad de Popayán entre el 19 y 20 de abril de 2007.
} 


\section{Introducción}

\section{¿La Educación Física en crisis?}

La Motricidad Humana sin Educación Física no tiene Historia, pero la Educación Física sin Motricidad Humana no tiene futuro (Sergio, 1987).

La contundente frase de Manuel Sergio (1987) en su tesis doctoral, puede convertirse en una premonición que permita hacer visible hitos históricos trascendentales para el proceso de constitución de un nuevo campo de saber a costa de la Educación Física y /o promover la transformación o cualificación de ésta. Pero ¿qué significado tiene esta frase para los profesionales relacionados con el campo de la Educación Física? ¿Qué relación tiene esta propuesta con la Ciencia de la Acción Motriz de origen francés? (Parlebas, 1992), donde la Educación Física es considerada como una pedagogía de las conductas motrices y parte de esta ciencia.

¿Quedará la Educación Física subsumida por esta visionaria propuesta? ¿Se trata de una nueva especie de ciencia madre?, o más bien ¿se intenta realizar un desplazamiento del concepto de Educación Física, a partir de un enfoque omnicomprensivo, similar al realizado por el concepto anglosajón de Currículo, para desplazar los conceptos de pedagogía y didáctica? (Contreras, 1998: 24).

La verdad es que en nuestro medio se conocen propuestas como las del alemán Ommo Grupe (1976), donde a partir de una visión amplia de la Pedagogía se considera que la Educación Física (ejercicios físicos, juego y deporte) debe hacer parte de las "Ciencias de la Educación"2, postura que es ratificada y complementada directamente por autores españoles contemporáneos como Rodríguez (1997: 217) y Sáenz y López, (1997: 98)

e indirectamente por nociones como la de Cultura Física promovida por los antiguos países socialistas, donde se incluyen como manifestaciones de esta "la Educación Física, la recreación, y el tiempo libre, el deporte como fenómeno social, la Actividad Física Laboral y la Actividad Física como medio de Rehabilitación, entre otras" (Consejo de Instituto de Educación Física y Deporte de la Universidad de Antioquia, 1988).

Sin embargo existen otras propuestas que intentan darle un carácter científico a las prácticas de la Educación Física, pero alejándose de una ciencia tradicional (en este caso de la Pedagogía) y tratando de constituir

\footnotetext{
2 En la actualidad este concepto en Colombia es considerado como uno de los paradigmas pedagógicos provenientes de la cultura francesa a partir de los trabajos del Grupo de Investigación Historia de la Práctica Pedagógica.
}

una ciencia autónoma, es el caso de la propuesta alemana de Ciencia del Deporte (movimiento, juego y deporte) (Haag, 1994), que retoma y continúa los trabajos de Diem (1953) y las Ciencias de la Actividad Física y el Deporte (Ruiz, 1995).

Pero, ¿cómo ha sido en Colombia el proceso de constitución de las prácticas de la Educación Física, como campo de saber y de poder? Para responder a esta pregunta, se hace necesario acudir a la Historia crítica de la Educación Física en nuestro país, para ello se toman como referencia algunos de los trabajos investigativos realizados en Colombia desde el enfoque de investigación arqueológico, teniendo como hilo conductor la investigación en la que se hace visible una problematización que es recurrente en la Historia de la Educación Física colombiana, como es la de la "oposición entre los discursos de la Educación Física y el Deporte entre 1968 y 1991" (Pinillos, 2004).

Para ello inicialmente es importante mencionar que los profesores de la Facultad de Educación Física de la Universidad Pedagógica Nacional de Bogotá han liderado la visión de la Educación Física como "un subproceso del sistema general de Educación [...] que usa juegos, deportes, danza, gimnasia y otras actividades de movimiento como medios pedagógicos de aprendizaje y de Educación" (1985), apoyándose en visiones de la Educación como la teoría de la Psicomotricidad de origen francés. (Velásquez, 1974). Representan esta visión profesores como Bonilla (1979, 1985, 1986,1988, 1989, 1991), Morales (1990), Camacho $(1985,1990)$, Chinchilla (1989), entre otros.

Por otro lado, en Colombia la "Ciencia del Deporte" se convirtió en el discurso con el que se pretendió darle mayor cientificidad a la Educación Física, a la Recreación y al Deporte, aprovechando la asesoría de los alemanes en estos campos entre 1974 y 1984

Aún así se podría decir que la nueva constitución colombiana recibe un campo de saber en medio de una crisis "epistemológica" y "profesional", pues si bien su escenario central ha estado en la institución escolar, o mejor en términos actuales, en la Educación formal, lo cual por lo menos le permite compartir una identidad disciplinar de la mano de la Pedagogía, su objeto se complejiza más cuando entre finales de la década de los ochenta y comienzos de la década de los noventa en la formación de profesionales se diversifican sus prácticas, acontecimiento que se evidencia en el plan de estudios del Instituto de Educación Física de la Universidad de Antioquia, que sin renunciar a la titulación de Licenciado en Educación: Educación Física, Recreación y De- 
porte, permite que sus estudiantes realicen su práctica profesional en campos que se pueden denominar como: Educación física para todos; Educación Física y Educación Especial; Educación física y Salud; Educación Fisica y Entrenamiento Deportivo, Educación Física y Recreación, Educación Física y Expresiones Artísticas (Pinillos, 2004: 297).

Esta diversificación de los campos de acción de la Educación Física, que ya había sido objeto de análisis en el III Congreso Nacional de Educación Física en 1988, en relación con el Deporte genera preocupaciones que confirman la oposición entre la lógica de la Educación Física y la lógica de esté a saber.

Entre otros tantos problemas el III Congreso Colombiano de Educación Física señala como erróneo el antagonismo que se pretende formar entre Formación y alto rendimiento, etc., hecho que obedece al empirismo $\mathrm{y}$ al eclecticismo. En consecuencia, llama al estudio riguroso y a la generación de prácticas innovadoras que construyan alternativas a estas barreras dispersantes" (Asociación Colombiana de Profesores de Educación Fisica, 1989: 30).

Este acontecimiento de la "diversificación de las prácticas", se convirtió en un reto para la educación fisica, tanto a nivel disciplinar como profesional, pero al mismo tiempo amplió la crisis sobre el objeto de estudio $^{3}$ y la oposición entre la educación física y el deporte, la cual se transforma en una oposición entre la pedagogía y los demás campos de acción que emergen, debido a que se considera que la pedagogía es propia sólo del campo de acción de la educación formal o docencia escolar.

\section{En busca del proceso de constitución de la Educación Física en Colombia}

Un viaje muy somero por la génesis de la Educación Física en Colombia con la ayuda de los resultados de la investigación en mención (Pinillos, 2004), nos permite visualizar que desde los discursos, más que desde las prácticas, esta no encuentra su norte ni en la gimnasia, ni en el deporte, quizás por su pretensión no sólo de atender una dimensión del ser humano, sino también otras. Lo cual complejiza su objeto de estudio.

Según los documentos analizados, se puede apreciar que en Colombia a mediados de la década de los setenta y la de los ochenta, el intento de desplazar la noción de

La diversificación de las prácticas de la Educación Física es anunciado como uno de los aspectos que genera la crisis de la Educación Física en el contexto global (Parlebas, 1992) educación física, por la de ciencia del deporte, con la pretensión de cientificidad, termina relegando a ésta a un papel instrumental, supeditada a los fines utilitarios del rendimiento deportivo, sin atisbo de aspectos formativos o pedagógicos, aspecto que se hace visible en las oposiciones que emergen a nivel de los sujetos, las instituciones y los saberes.

Como reacción a esta propuesta se genera una reflexión en el país que se puede caracterizar por la búsqueda de identidad disciplinar y profesional y la producción académica en este campo de saber, donde se destaca el contradiscurso que se elabora en Colombia para oponer resistencia a la tendencia mundial de la ciencia del deporte.

Es así como la reforma del sistema educativo del país en la década de los setenta denominada Renovación Curricular (Ministerio de Educación Nacional, 1976, 1978), se presenta como una oportunidad para repensar la educación física con el fin de elaborar los programas curriculares para la enseñanza de la educación física en la educación formal, donde entran en pugna la visión de la educación física, desde el punto de vista de la apropiación de los discursos de las ciencias del deporte de origen alemán, y la apropiación de la psicomotricidad, de origen francés a manera de contradiscurso, pudiendo sin embargo, finalmente, convivir ambos paradigmas bajo la denominación de área de la educación física, la recreación y el deporte (Ministerio de Educación $\mathrm{Na}$ cional, 1984).

Sin embargo, por fuera de la Institución escolar el concepto de deporte es el macroconcepto que se utiliza para abarcar los sistemas de la Educación Física, el Deporte y la Recreación en lo que se denominó la Ley Marco del Deporte (Ministerio de Educación Nacional, 1984), lo cual fue retomado también por la actual Ley del Deporte (1995).

Se destaca en este recorrido histórico en busca de los fundamentos epistemológicos de la Educación Física, un acontecimiento en la Universidad del Valle donde, a partir de 1987, comienza a gestarse una visión de la educación física, que centra su atención nuevamente en el cuerpo, como objeto primigenio de la Educación Física y lugar donde confluyen todos los discursos de ésta. En efecto, se volvía al cuerpo con la intención de conocer las condiciones que hicieron posible la emergencia de la noción de educación física, de reconocerla como una práctica pedagógica y reconocer su historicidad (Quesada y Sánchez, 1991). Reflexión que se puede constituir en la gestación de una nueva visión de la Educación Física, como veremos a continuación. 
Es así como la década de los noventa en Colombia se inicia con una nueva Constitución, que desde una concepción más liberal, o para algunos, más de corte "izquierdista", deja plasmado el sueño de diferentes sectores del país relacionado con la construcción de una nueva sociedad y un nuevo ser humano, lo cual le exige al país una transformación de tipo económico, político y cultural. Se prevé que esta transformación será muy lenta, debido a las enseñanzas que nos dejara una constitución de corte más conservador por espacio de más de 100 años (1886-1991).

La Educación se convierte así en el eje central para el desarrollo del nuevo proyecto político nacional, para ello el nuevo sistema educativo que integra a la Educación formal, la no formal y la informal tiene la gran responsabilidad de construir "proyectos educativos institucionales", con la participación de la "comunidad educativa", la cual incluye no sólo los directivos escolares, estudiantes y profesores, sino también a los padres de familia y representantes del sector productivo.

El Proyecto Educativo Institucional se convierte entonces en una estrategia administrativa con la que se pretende articular, a partir de los "proyectos pedagógicos", "programas" derivados de "planes de desarrollo", tanto del orden nacional, departamental, como municipal, asi como de diversas instituciones y sectores del país, con el propósito de hacer posible el nuevo proyecto de nación.

\section{La Motricidad humana, objeto de estudio, desde la perspectiva de la Educación Física Sistémica}

En la historia de la Educación Física contemporánea en Colombia, el movimiento corporal ha sido considerado como el objeto de estudio de ésta (Ministerio de Educación Nacional, 2000), atendiendo a planteamientos de teóricos como el de Kurt Meinel, representante de la antigua República Federal de Alemania y L.P. Matiejev de la antigua Unión Soviética, los cuales ingresaron al país gracias a los intercambios realizados con Cuba, desde finales de la década de los setenta

Sin embargo, con la asesora ofrecida por los alemanes, teniendo como referencia las nuevas teorías de aprendizaje, se intenta superar el concepto de "movimiento corporal", con el de "sensomotricidad", con el fin de integrar pensamiento y acción; concepto que a su vez tiene mucha cercanía con el de psicomotricidad de origen francés, este último representaba un paradigma en el campo de la Educación en general, liderado por los representantes de la psicología del desarrollo.
De acuerdo con lo anterior, el movimiento representa para muchos autores contemporáneos, una noción que da cuenta de un aprendizaje de tipo mecánico, inconsciente, es decir conductista; mientras que nociones como sensomotricidad, psicomotricidad y motricidad representan un aprendizaje consciente y creativo, de orden constructivista. Incluso en la actualidad desde la perspectiva cognitivista se propone un nuevo concepto el de "competencia motriz" (Ruiz, 1995).

El Instituto de Educación Física de la Universidad de Antioquia en la década de los noventa, se puede considerar como la cuna donde se empieza tejer con mayor consistencia, pero también con mayores dificultades, una nueva visión de la educación física sustentada teóricamente en la pedagogía y las demás ciencias humanas y sociales, así como también, supuestamente, en una visión pedagogizada de las ciencias naturales, que toma una versión humanizada del concepto de Motricidad (Castañer y Camerino, 1996) como objeto de estudio y se empieza a implementar en el 1999 desde una estructura curricular novedosa, apoyada en los enfoques curriculares de tipo "crítico" y "práctico" (Franco et ál., 2000).

Esta nueva visión de la Educación Física tiene su génesis en acontecimientos como el "semestre taller", nombre dado en 1992 a un movimiento estudiantil que generó una estrategia académica y administrativa temporal (un semestre académico), para atender en sus términos "la emergencia sanitaria", por la que pasaba la formación de los profesionales de la educación física en la Universidad.

La nueva visión de la educación física que se ponía en juego, en un currículo caracterizado por la "problematización del conocimiento" (Pinillos, 2003), se convirtió en una disculpa para seguir pensando en una Educación Física que, como concepto macro, pretendía dar cuenta de campos de acción profesional relacionados con el sector de la educación, la salud, el deporte y la recreación, los cuales articulados a un objeto común denominado Motricidad buscaban aportar a la construcción de una nueva sociedad y al mejoramiento de la calidad de vida, finalidades que de manera explicita o implícita hacen parte del nuevo proyecto político nacional.

Este nuevo enfoque curricular de la educación física, se irradió con diferentes grados de intensidad a otras universidades del país y a varias instituciones de la educación básica y media, así como a algunas instituciones gubernamentales. A partir de esta experiencia en el departamento de Antioquia se pueden resaltar varios aspectos positivos, sin embargo también deja muchos aspectos por mejorar, aspectos que dan cuenta de la 
forma como fue apropiado el nuevo objeto de estudio propuesto para la Educación Física.

\section{Conceptos emergentes para la construcción} de una episteme de la Educación Física alrededor de la Motricidad en la perspectiva del desarrollo humano en Colombia

A pesar del desencanto, que acompaña a un sector de la comunidad educativa, que reclama insistentemente, las prácticas, sobre todo las de tipo deportivo, pues al parecer de muchos, con el nuevo currículo no se pretende formar educadores físicos, sino filósofos y pedagogos, quizá tengan razón. El proyecto pedagógico de Educación Física de la Universidad de Antioquia, desde el punto de vista de la "reflexión epistemológica", se ha convertido en una condición de posibilidad para la construcción de una episteme de la educación física alrededor de la Motricidad en la perspectiva del desarrollo humano en Colombia.

En tal sentido se han ido configurando una serie de "filiaciones" entre conceptos a la manera de Canguilhem (1971). o "articuladores", desde la perspectiva foucaultiana (1999), a partir de los resultados de las investigaciones de los grupos de Investigación: Calidad de la Educación Física, hoy denominado Ocio, Expresiones Motrices y Sociedad, Cultura Somática y más recientemente Estudios de Educación Corporal, los cuales se pueden denominar como "corrientes" de una tendencia que nace en la educación física y que orienta sus búsquedas en la perspectiva del desarrollo humano, utilizando como medio la motricidad humana y el ocio, entendidos como necesidades humanas; propósito con el que se encuentran también con el grupo de investigación interuniversitario de Motricidad y Mundos Simbólicos, también colombiano, entre otros.

\section{De la pedagogía motriz a la pedagogía del cuerpo}

Los primeros conceptos contemporáneos que nos aproximan a una episteme de la educación física alrededor de la motricidad tienen que ver con el concepto de pedagogía motriz, debido a la importancia que se le da en nuestro país, por parte de un sector de la comunidad académica, al concepto de pedagogía desde una visión humanista y a la redimensión del concepto de motricidad.

Este concepto tiene su génesis en acercamientos de los grupos de investigación mencionados, relacionados con la pedagogía del cuerpo y la pedagogía de las expresiones motrices (et ál., 2005).

Por pedagogía motriz se entiende un "proceso educativo fundamentado en el desarrollo de las conductas motrices, para que junto con las demás esferas del ser humano le permitan construir una identidad particular y universal (cultura)" (Moreno et ál., 1995). Se trata de una visión de la educación física que se apoya en planteamientos de autores como el francés Pierre Parlebas, que aborda la totalidad del ser humano, teniendo como punto de partida la dimensión motriz, como es expresado a continuación:

Hoy la pedagogía motriz intenta asumir de hecho el problema de la Educación integral. Se requiere de otro maestro con un dominio de un modelo pedagógico y didáctico específico, que aborda el hombre para mejorarle integralmente desde una pedagogía de las conductas motrices, que plantee procesos de investigación al interior de la escuela y su formación en valores a los nuevos ciudadanos que requieren este país (Moreno et ál, 1995).

En esta misma línea, Díaz y otros consideran a la Educación Física como un medio ideal para desarrollar otros aspectos del ser humano como es expresado en apartes de su trabajo de grado:

La educación física desde su aspecto formativo que implica también lo valórico y lo cognitivo es uno de los modelos propicios para el desarrollo psicosexual del escolar por las características de practica al utilizar el cuerpo como canal de comunicación, proyección y realización humana (1996: 82).

Desde otra mirada, la profesora Rubiela Arboleda, apunta a la necesidad de pensar en la relación entre Pedagogía y Cuerpo en los siguientes términos:

Los lineamientos definidos en las políticas educativas respecto al cuerpo constituyen una normatividad que lejos esta de asumir el sentido del cuerpo; esto es, la corporeidad como un elemento sustantivo en la formación integral de los individuos. El cuerpo ha sido limitado al campo motriz, llámese Educación Física, Cuerpo, Teatro, Recreo, entre otros -de lo que se infiere que se ha descorporeizado el denominado terreno intelectual- que si bien ofrece profundas alternativas pedagógicas, no justifica la eliminación del cuerpo en otras esferas (Arboleda, 1997: 83).

Los trabajos posteriores de la profesora Arboleda desde el grupo de investigación Cultura Somática del Instituto de Educación Física de la Universidad de Antioquia le han permitido profundizar en la relación cuerpo, educación, educación física y sociedad desde 
una perspectiva interdisciplinaria, apuntándole a la construcción de una pedagogía del cuerpo.

Por otro lado, el profesor Pinillos, refiriéndose a la formación de profesionales en el campo de la Educación Física, habla de la importancia de una Pedagogía y Didáctica de la Educación Física, la cual tiene por objeto construir "un hilo conductor que permita la conexión entre los contenidos relacionados con las características generales del ser humano, las condiciones de su entorno ecológico y su objeto de estudio [...] la dimensión motriz del ser humano" (Pinillos, 1999).

Por su parte el profesor Alberto Echeverri propone que en el "dispositivo formativo comprensivo" (1996) de las Escuelas Normales Superiores asesoradas por la Facultad de Educación de la Universidad de Antioquia se aborde el núcleo de Pedagogía del Cuerpo, como un espacio alrededor del cual los maestros reflexionen sobre las áreas de educación artística y la educación física, la recreación y el deporte desde la perspectiva de la formación de profesores. De esta manera en la Normal Superior Envigado se dice lo siguiente:

La educación en pedagogía del cuerpo es un proceso pedagógico organizado dirigido a la formación de los alumnos maestros, con capacidad de percibir, sentir, comprender y evaluar la belleza en la realidad circundante, en la vida social, en el trabajo y en el arte. Sus relaciones con la educación intelectual son muy estrechas, amplía el horizonte intelectual de los alumnos-maestros, contribuye a comprender y valorar mejor la esencia de los fenómenos, desarrolla la capacidad de observación, y la percepción más clara del mundo que les rodea.

Es de gran importancia la vinculación de lo estético con la educación física, en lo que se refiere a la belleza en el aspecto externo del educando, a la elegancia y gracialidad de los movimientos, como a la estética de sus gestos y modales. La gimnasia rítmica desarrolla hermosas cualidades como la disciplina, la cortesía y el comportamiento. Además, toda actividad deportiva llevará al desarrollo de las valencias físicas: fuerza, velocidad, equilibrio, coordinación, resistencia, flexibilidad y elasticidad (Velásquez et ál., 2004).

Llama la atención la articulación de estos dos campos de saber en torno a la Pedagogía, se puede tratar de una alianza estratégica en torno al concepto de cuerpo que aporte buenos resultados.

\section{Pedagogía de la Motricidad: una mirada desde el desarrollo humano y la promoción de la salud}

Este giro de los fines de la educación física en Colombia, orientado hacia la formación de la totalidad del ser humano, es asumido en Colombia desde el concepto contemporáneo de desarrollo humano, otro concepto que se ha desarrollado a la par con el concepto de pedagogía, entendiéndose como un saber que tiene por objeto el estudio de las teorías relacionadas con la formación del ser humano, uno de los objetos de la Pedagogía. En este sentido en el Instituto de Educación Física de la Universidad de Antioquia, teniendo como ejes, la teoría de sistemas y el pensamiento complejo, entre otros, se ha profundizado en el estudio e visiones como la del desarrollo a escala humana, planteada por Max Neef (1997) y otras, visiones de la calidad de vida, como las de Amartya Sen (1993), las esferas del Desarrollo Humano de Roldán (1997); las metas del desarrollo humano de Ramírez et ál. (1997) y las capacidades humanas de Weinstein $(1978,1995)$. Ello con el fin de establecer una relación entre éstas y la Motricidad, entendida como una de las dimensiones del ser humano y el objeto de estudio de la educación física.

En la apuesta por el desarrollo humano desde la perspectiva de la Motricidad, se destaca la investigación: Las metas del desarrollo del niño y la niña por medio de la familia en los municipios de Guatapé y Alejandría del Departamento de Antioquia, realizada desde la línea de investigación Motricidad, Desarrollo Humano y Promoción de la Salud, del grupo de Investigación Calidad de la Educación Física, la investigación de corte cualitativo, "presenta una mirada de la promoción de la salud como estimulo de potencialidades por medio de las metas del desarrollo. Además, toma como dispositivo fundamental la mirada hacia las capacidades humanas centrada en la transformación del sujeto y desde allí, a las posibilidades de transformación social y cultural" (Uribe et ál., 2004). Para ello buscan los sentidos que las familias le otorgan al desarrollo humano apoyándose en la visión del desarrollo humano liderada por médico antioqueño y profesor universitario Humberto Martínez (1997) y la visión del desarrollo humano, desde las capacidades humanas de Weinstein (1995). Se destaca en esta investigación el análisis sobre los sentidos que las familias le 
otorgan a "las formas expresivas de la motricidad", tomando como punto de partida la apropiación del concepto de Motricidad de Manuel Sergio (1999).

Posteriormente el mismo profesor Uribe, en compatila de otros profesores miembros del grupo de investigación en mención ${ }^{4}$, realizan una investigación desde el enfoque crítico social denominada "la Pedagogía de la Motricidad como estrategia para la promoción de la salud", con la que obtienen el titulo de Magíster en salud colectiva, en la Facultad de Enfermería de la Universidad de Antioquia. En esta investigación se entiende el concepto de pedagogía de la motricidad como "la manifestación del ser humano a través de su expresión motriz intencionada hacia la potenciación de las capacidades humanas [...] que comprende en su estructura fundamental la interacción de las dimensiones introyectiva (relación consigo mismo), extensiva (relación con el mundo objetual), y proyectiva (relación con los otros) del cuerpo como proceso dinámico y permanente orientado a la formación de sujetos individuales y colectivos" (Uribe et ál., 2004: 66).

El desarrollo histórico de la educación física y el reconocimiento de los factores determinantes de su posición social le permiten a estos profesores avanzar hacia una interpretación de la educación física como una pedagogía de la motricidad que reivindique la orientación pedagógica del saber y su compromiso con la potenciación de las capacidades humanas para superar visiones fragmentadas de la persona, del mundo y de la vida.

Con la pedagogía de la motricidad se busca, a partir de las expresiones motrices, brindar más y mejores oportunidades de ser, hacer, vivir y compartir como humanos, desde una mirada de carácter interdisciplinar, transdisciplinar.

La pedagogía de la motricidad como intención formativa aborda la reflexión sobre los procesos educatiwos, el compromiso con un concepto de hombre y de sociedad y con la construcción de conocimiento. En este propósito asume la motricidad como una expresión y manifestación cultural del proceso individual y colectivo de formación y cualificación del ser humano desde su dimensión motriz en interacción con otras.

En esencia, con este enfoque se busca generar espacios y alternativas para potenciar el desarrollo humano y social a partir de la motricidad como dimensión confgurativa de éste.

Se hace referencia a Víctor Alonso Molina Bedoya, Elkin de Jesús Vergara y Melquised Blandón Mena.

\section{De la motricidad humana}

\section{a las expresiones motrices}

Las expresiones motrices emergen como un concepto que se ha ido decantando gracias, por un lado a la apropiación de concepto contemporáneo de motricidad, tomando como referente central la propuesta de los españoles Castañer y Camerino (1996), y por otro lado a la apropiación del concepto de cultura somática de los alemanes.

El concepto de expresiones motrices es entendido inicialmente como "los usos del cuerpo que pasan por lo operativo y tocan con el sistema de significaciones, las percepciones, las actitudes, las prácticas y las representaciones individuales y colectivas del mismo, en un contexto sociocultural" y hace parte de una de las dimensiones de la Cultura Somática (Correa, 2001).

En relación con la apropiación de la propuesta española, ésta se concretó inicialmente en el proyecto de cualificación de maestros denominado Microcurrículo en Pedagogía Motriz (Moreno et ál, 1995), en el cual se utilizaron como ejes temáticos de los módulos El saber en sí: motricidad básica (actividades atléticas, gimnásticas, acuáticas y luctatorias), motricidad específica, motricidad gestual y juegos con pelota; Elementos constitutivos de la motricidad: capacidades perceptivo, físico y sociomotrices; y Enseñabilidad: desarrollo motriz y aprendizaje motriz. Es importante señalar que el concepto de motricidad básica y específica estaba muy emparentado con el de habilidades motrices básicas y específicas de los autores en mención.

Podría decirse que una segunda apropiación de la visión sistémica de la motricidad planteada por estos españoles tiene lugar en el nuevo proyecto curricular del Instituto de Educación Física de la Universidad de Antioquia (1999), es así como el "núcleo problémico", denominado "motricidad", se estructura a partir de cuatro componentes: Epistemología de la motricidad; Estructuras básicas y fundamentales; Expresiones motrices; y Pedagogía de las expresiones motrices. Estos componentes pretenden en su orden servir de guía para reflexionar sobre el proceso de constitución de la educación física, sus conceptos articuladores, sus campos de acción y la pedagogía y didáctica de éstos.

Es bueno acotar que las expresiones motrices que se nominaron inicialmente en este proyecto curricular fueron: Expresiones motrices básicas, artísticas, deportivas; Expresiones motrices y lúdica. Por otro lado, los énfasis relacionados con los campos de acción de la educación física pensados tenían que ver con en el sector escolar, el deportivo y la "motricidad comunitaria", concepto rela- 
cionado inicialmente con prácticas de ocio y la salud, en poblaciones no escolarizadas.

Una tercera apropiación del concepto la realiza el profesor Pinillos (2002), quien de la mano de la propuesta de Castañer y Camerino (1996) y siguiendo a Ruiz (1995) dice lo siguiente:

propongo la denominación de competencia motriz como una competencia adecuada para evaluar, conocimientos relacionados con las capacidades de corporalidad, espacialidad, temporalidad, fuerza, resistencia, velocidad, flexibilidad, expresión corporal y la lúdica así como patrones básicos de movimiento, habilidades motrices básicas y habilidades motrices específicas de tipo deportivo, dancístico entre otras. Alejándome un poco de la propuesta del Ministerio de Educación Nacional (2000) para el área de la Educación Física la recreación y el deporte que bajo la denominación de "competencia praxiológica" plantean procesos o dominios de contenidos tales como: desarrollo físico y motriz; organización del tiempo y del espacio; formación y realización técnica; interacción sociocultural; expresión corporal; recreación; y experiencia lúdica.

Es así como partiendo de las dimensiones de la Motricidad Humana (Castañer y Camerino, 1996), o sea de las diferentes acciones que es capaz de realizar el ser humano, se proponen como competencias generales para el área de educación física el conocimiento corporal, la formación corporal y la expresión corporal. Para cada una de las competencias se establecen los siguientes niveles y desempeños: nivel 1: reconocimiento de capacidades y habilidades motrices; nivel 2: interpretación y uso de las capacidades y habilidades motrices; y nivel 3: producción y generalización de expresiones motrices.

Una aproximación contemporánea al concepto de motricidad como génesis del concepto de Expresiones Motrices en Colombia, es presentada por la profesora Rubiela Arboleda, en una conferencia ofrecida a un equipo de trabajo interdisciplinario, en ella se refiere a la motricidad en los siguientes términos:

Se reconoce en el discurso de la motricidad una búsqueda de la comunidad académica local e internacional por una episteme propia de la Educación Física, así la motricidad se identifica como una dimensión que configura al sujeto y se proyecta al escenario de lo social. La motricidad es la expresión de la subjetividad, es comunicación del ser humano de su voluntad, creatividad, propositividad, intencionalidad y sensibilidad (Arboleda, 2002: 179).
Al decir de la profesora Arboleda, la motricidad evoca la corporeidad, entendida ésta como la conciencia del cuerpo que integra su reconocimiento y vivencia.

La versión más actualizada del concepto de expresiones motrices fue presentada por la profesora Arboleda en el Fórum Barcelona 2004, en el marco de la temática El Deporte: diálogo universal, donde se refiere a éste en los siguientes términos:

Como consenso académico temporal he propuesto la denominación Expresiones Motrices, con la que se hace referencia a aquellas manifestaciones de la motricidad que se realizan con distintos fines; lúdicos, agonístico, estético, preventivo, de mantenimiento, de rehabilitación y de salud entre otros, organizadas siguiendo una lógica interna, que establece un código legitimado en un contexto social, y por el cual se admiten y/o prohíben unos gestos, se ofrecen pautas de comportamiento, se crean actitudes y se promueven creencias. Éstas privilegian ya no sólo el código gestual observable, repetitivo e instrumental, sino las intenciones subjetivas puestas en juego en el acto mismo, lo que les otorga un significado importante en la comprensión de su potencial pedagógico. El concepto de expresiones motrices es bastante amplio, dado que admite técnicas corporales: danza, teatro, hidrogimnasia, juegos, etc., que quedarían excluidas al utilizar como término genérico el de deporte que, como será usado en este caso, es asociado a competencia, codificación, rendimiento, reglamento y con ello a ciertas disciplinas formalmente establecidas. El deporte así entendido hace parte de las expresiones motrices y esta matizado por el sentido agonístico (Arboleda, 2005: 92).

Entendida la motricidad como el objeto de estudio de la educación física, se puede inferir que la expresiones motrices se constituyen en un concepto que pretende dar cuenta de los diferentes campos de acción de la educación física en la perspectiva del desarrollo humano en Colombia.

\section{El Ocio: una dimensión del ser humano}

El concepto de ocio es introducido a la Universidad de Antioquia, por parte de Fernando Tabares (2000), candidato a doctor en Ocio y Desarrollo Humano en la Universidad de Deusto en el País Vasco, quien desde la Corporación Civitas y en compañía de otros profesores de la línea de ocio y tiempo libre del grupo Ocio, Expresiones Motrices y Sociedad, lidera una nueva visión 
del ocio, como necesidad humana, que requiere de unos satisfactores particulares, de acuerdo a nuestro contexto histórico y social, apoyándose en autores como Cuenca (1997) y Max Neef et ál (1997). Se trata de una visión supremamente interesante, porque no sólo aporta al episteme de la Educación Física, sino que intenta marcar dlaramente los límites entre estos dos campos de saber.

Según Molina y et ál (2001),

$\mathrm{El}$ ocio se puede comprender como tiempo libre de trabajo, actividad elegida con cierta libertad, un estado mental, un conjunto de actividades y uso de tiempo. Pero lo cierto es que el ocio no puede ser entendido exclusivamente como espacio o actividad, porque siendo algo constitutivo del ser humano, es su humanidad en la dimensión más pura; por ello existe en todas las culturas y en todos los seres humanos de todos los países del mundo, sin importar su desarrollo industrial o tecnológico, ni su posición geográfica, ni su raza, credo o ideología. El ocio es inherente al ser humano, y por lo tanto, no es una finalidad ni una consecuencia, como se pretende, sino que es un elemento inherente a las personas y a las sociedades como elemento potenciador (175-176).

Se trata de una capacidad humana que por las caracteristicas de las prácticas de la educación física contrisuye a articular la episteme de la educación física por medio de un dialogo entre la motricidad y el ocio, como capacidades humanas y el concepto de expresiones motrices, como un concepto que desde la perspectiva de la educación física, integra estos dos campos de saber en gro del desarrollo humano.

\section{La pedagogía, un reto para la humanización de la educación física}

La pedagogía puede ser entendida como un campo de saber propio de los maestros, que tiene como objeto la reflexión sobre la educación, práctica a la cual se le asigna la responsabilidad social de la formación del ser humano y la enseñanza de las ciencias, de acuerdo a unas condiciones sociohistóricas determinadas. Al igual que en la mayoría de las ciencias sociales el discurso educatino está conformado por diversidad de campos, objetos y problemas de investigación frente a los cuales no hay un acuerdo entre la comunidad disciplinar (Pinillos, 2004).

Dentro de los problemas que debe atender este campo de saber en la actualidad se pueden mencionar los siguientes:
1. Naturaleza disciplinaria de la Pedagogía; 2. La distinción entre Didáctica y Pedagogía; 3. Articulación entre teoría y experiencia; 4 . Debate en torno al ser intelectual del maestro y los investigadores; 5. Las disputas entre la formación de docentes y la participación en ellas de la ciencia y la Pedagogía (Echeverri, 1996: 90).

En el transcurso de la historia de la práctica pedagógica en Colombia, se puede distinguir la apropiación de modelos como el Lancasteriano, Pestalozziano, Católico, la Escuela Activa, la Tecnología Educativa, el Constructivismo y el Cognitivismo. Es importante resaltar que la producción académica en este campo ha llevado a que se realicen investigaciones donde se han producido conceptos como el de "campo intelectual de la Educación" (Díaz, 1993) y a partir de éste el de "campo pedagógico" (Echeverri, 2001). Entendido este último como "el que posee mayor potencia para conjugar y recibir las concepciones y propuestas que caracterizan la pedagogía contemporánea. La potencia del campo esta dada por su capacidad de alojar los conceptos, problemáticas y preguntas que se formulan en la actualidad desde ciencias y disciplinas diferentes a la pedagogía, así como desde los diferentes paradigmas: ciencias de la educación, pedagogía y currículo" (Rodríguez, 2002: 129).

Por último es bueno mencionar la redimensión que se le ha dado a la noción de "práctica pedagógica", a partir de investigaciones realizadas por espacio de 30 años aproximadamente por parte del Grupo de Investigación Historia de la Práctica Pedagógica, utilizando la "caja de herramientas" que ofrece el enfoque de la historia crítica de Michel Foucault. Para ellos la práctica pedagógica abarca los siguientes aspectos:

- Los modelos pedagógicos tanto teóricos como prácticos utilizados en los diferentes niveles de enseñanza.

- Una pluralidad de conceptos pertenecientes a campos heterogéneos de conocimiento retornados y aplicados por la Pedagogía.

- Las formas de funcionamiento de los discursos en las instituciones educativas donde se realizan prácticas pedagógicas.

- Las características sociales adquiridas por la práctica pedagógica en las instituciones educativas de una sociedad dada que asigna unas funciones a los sujetos de esa práctica.

- Las prácticas de enseñanza en diferentes espacios sociales, mediante elementos del saber pedagógico $(\mathrm{Zu}-$ luaga, 1987: 5). 
Vista la pedagogía de esta forma, considero que ha sido esta la que de manera explícita o implícita ha permitido la articulación entre los conceptos a los que se ha hecho alusión, con el fin se servir de soporte epistemológico a la educación física

Si bien la pedagogía, hecha visible más en los discursos de los maestros encargados de la formación de profesionales del campo de la educación física, que en los maestros que se desempeñan en la educación básica (Pinillos, 2004), como se hace visible en los resultados de las investigaciones realizadas por Pinillos et ál. (2005) y estudios de educación corporal (2005). Este campo de saber emerge como una condición de posibilidad, para la constitución de una episteme ${ }^{5}$ de las prácticas de la educación física en la educación formal y probablemente también en los nuevos campos de acción que se perfilan. Al respecto se decía en el mencionado Congreso de 1988:

La educación física no puede renunciar al deporte de rendimiento, ni a la competencia, pues poseen valores inmensos para el mejor logro del papel de la Educación Física en el mundo. Debe luchar por reorientar el Deporte de rendimiento dentro de los lineamientos pedagógicos que lo originaron, preparando para ello convenientemente los profesionales que la administren con lujo de competencia y adoptando unas posiciones decididas que le permi$\tan$ reasumir su responsabilidad ante la comunidad (Ramos, 1989: 34).

Esto no quiere decir tampoco, según los datos revelados por varias investigaciones, que el mayor porcentaje de asignaturas en la formación de profesionales esté orientado hacia los deportes, ni que la educación física deba tener como único fin la promoción y enseñanza del deporte. En este sentido, el reto mayor para la educación física lo constituye pedagogizar el deporte o, en términos más claros, humanizar el deporte, convirtiéndolo realmente en un medio de la educación, como tanto se promulga, sólo que sus prácticas demuestran lo contrario.

Sin embargo, una de las conclusiones de la comisión del Congreso de 1988 encargada del tema de la formación profesional del profesor de Educación Física dice que en las instituciones formadoras existe una "subestimación y limitación conceptual de lo pedagógico", lo cual sin temor a expresarlo se podía extender a nuestros días.

\footnotetext{
5 A partir de los procesos históricos que configuran, entre otros, la filiación o articulación de los conceptos Pedagogía del Cuerpo, Pedagogía de la Motricidad, Expresiones Motrices y Ocio.
}

Al respecto el profesor Morales (1990), si bien reconoce que uno de los mayores avances en cuanto a trabajo teórico de la educación física tiene que ver con la teoría pedagógica, menciona que lo realizado hasta esa época "está centrado en un tratamiento didáctico de la disciplina, detectable en los pocos artículos que son publicados en el medio". Apreciaciones, que con raras excepciones también se pueden asumir como actuales. El reto entonces para constituir una episteme de la educación física alrededor de la motricidad en la perspectiva del desarrollo humano consistiría entonces en fortalecer el ámbito formativo de esta, casualmente el que más se le exige a las prácticas orientadas al rendimiento físico, es decir, el que tiene que ver con el proceso de humanización del ser humano.

Pero realmente ¿puede la pedagogía seguir siendo el referente, no sólo didáctico, sino humanista de la diversidad de prácticas relacionadas con la Educación Física? ¿Puede existir un referente teórico común? ¿Se puede mantener el mismo objeto de estudio? Es más, ¿sería la educación física la denominación común?

En la actualidad los trabajos del grupo de Investigación Ocio, Expresiones Motrices y Sociedad, apuntan a la construcción de una pedagogía de las expresiones motrices y a una pedagogía del ocio, a partir de la confluencia de teorías cercanas como las que tienen que ver con el desarrollo humano, la promoción de la salud, la motricidad y la pedagogía misma, con el fin de fortalecer, como se dijo con anterioridad, la tendencia que emerge en Colombia en la que inicialmente encuentra como aliados estratégicos a los grupos de investigación Cultura Somática y Motricidad y Mundos simbólicos.

Finalmente considero que en Colombia están dadas las condiciones de posibilidad, para que se empiece a hacer visible una episteme de la educación física alrededor de la motricidad humana y la pedagogía, probablemente, como una oportunidad para que la educación física en Colombia y en el mundo encuentre el norte epistemológico, pedagógico y didáctico, para contribuir al desarrollo humano y social.

Si analizamos lo que ha acontecido con la Historia de la Pedagogía, en la cual conviven en la actualidad el paradigma de la pedagogía y la didáctica; las ciencias de la educación y el currículo y las ciencias de la cognición, y teniendo en cuenta que algunos investigadores, están en la tarea de construir un campo conceptual y un campo aplicado que se adecue a las condiciones históricas actuales, pero sin perder la esencia de la pedagogía, es decir la formación del ser humano, considero que a partir 
de una visión de la Motricidad Humana, como objeto de estudio de la Educación Física, orientada al desarrollo humano, tenemos una tarea similar.

No puedo terminar sin expresar finalmente que en esta ponencia no hago referencia a la emergente ciencia de la Motricidad Humana, se trata de un análisis de tipo histórico que da cuenta de la apropiación contemporánea del concepto de motricidad como objeto de estudio

\section{Referencias}

Arboleda Gómez, Rubiela. (1997, julio-diciembre). Cuerpo y Pedagogía. En Revista Educación Física y Deporte. Universidad de Antioquia, Instituto Universitario de Educación Física y Deporte: Medellín, 19(2).

Arboleda, G. Rubiela. (2005). Las expresiones motrices en América Latina en la tensión global-local. Una apuesta desde la corporeidad, en el marco de la modernidad reflexiva. En: Revista Apunts. Educación Física y Deporte, 78. Barcelona.

Arboleda, G. Rubiela. (2002). La educación física: Un episteme alrededor de la Motricidad. En Parcelas y horizontes: un encuentro con las disciplinas. Grupos de Investigación. Bioantropología-Reproducción-Biogénesis. Medellín: Universidad de Antioquia.

Asociación Colombiana de Profesores de Educación Física. (1989, julio ). III Congreso Colombiano de Educación Física. Informe especial. En Revista Colombiana de Educación Física, 4. Bogotá: Asociación Colombiana de Profesores de Educación Física

Canguilhem, Georges. (1971). Lo normal y lo patológico. Buenos Aires: Siglo XXI.

Castañer, Marta y Camerino, Oleguer. (1996). La Educación Física en la enseñanza primaria. Barcelona: INDE.

Castañer, Marta y otros. (1996). La Educación Física en la enseñanza primaria. Barcelona: INDE.

Colombia. Ministerio de Educación Nacional. Decretos 088 de 1976 y 1419 de 1978 .

Colombia. Decreto 2845 de 1984. Ley Marco del Deporte. (1985, enero-diciembre). En Revista Educación Física y Deporte. Medellin: Universidad de Antioquia, Instituto de Educación Física y Deporte, 7(1-2).

Colombia. Ley 181 de 1995. Ley del. Deporte. Bogotá: Diario oficial n. ${ }^{\circ} 41.679$ del 18 de enero de 1995.

Colombia. Ministerio de Educación Nacional. (2000). Lineamientos curriculares: Educación Física, Recreación y Deportes. Áreas obligatorias y fundamentales. Bogotá: Cooperativa Editorial Magisterio.

Colombia. Ministerio de Educación Nacional. (1984). Marcos Generales del área Educación Física, Recreación y Deporte. Bogotá

Consejo del Instituto de Educación Física de la Universidad de Antioquia. (1999,25 de febrero). Diseño Curricular de programa de Licenciatura en Educación Física. Medellín. Resolución 002.

Consejo del Instituto de Educación Física y Deporte de la Universidad de Antioquia. (1988). Borrador de propuesta para la creación de la Escuela de Cultura Física en reemplazo del Instituto de Educación Física y Deporte. Archivo Instituto de Educación Física y Deporte de la Universidad de Antioquia. de la educación física, por parte de un sector de la comunidad académica en Colombia y sus perspectivas. En la actualidad, con mi vinculación a la Universidad del Cauca y la interacción con el grupo de estudio liderado por la profesora Eugenia Trigo, recogeré ${ }^{\text {argumentos }}$ para, en una próxima oportunidad, realizar un análisis de la relación entre la educación física y la ciencia de la Motricidad Humana. (D)
Contreras, Onofre. (1998). Didáctica de la Educación Física. Un enfoque constructivista. Barcelona: INDE.

Correa, Elvia y otros. (2001). Lineamientos curriculares para el área de la Educación Física, la Recreación y el Deporte. Medellín. Sin publicar.

Cuenca, M. (1997). Ocio y animación sociocultural: presente y futuro En Animación Sociocultural. Teorías, programas y ámbitos. Barcelona: Ariel.

Díaz, B., Restrepo, A. y Vélez, O. (1996). Educación Física y Educación Sexual: Una propuesta de lineamientos curriculares para el ciclo primario de Educación básica. Universidad de Antioquia. Facultad de Educación. Departamento de Educación avanzada. Tesis de grado para optar al título de Magíster en Psicopedagogía de la Educación. Medellín: Universidad de Antioquia.

Diem, C. (1953). Sportwissenschaft als Padagogik. En Die leibeserziehung.

Echeverri Sánchez, Jesús Alberto. (1996). Premisas conceptuales del dispositivo formativo comprensivo. En Revista Educación y Pedagogía, 8(16). Medellín.

Estudios de Educación Corporal. (2005). Grupo de Investigación. Sentidos de la Motricidad en el escenario escolar. Un inicio de rupturas paradigmáticas desde los actores de la Educación Física en Colombia. Medellín: Talleres Gráficos de Digital Express.

Foucault, Michel. (1999). La Arqueología del saber. XIX edición. México: Siglo XXI Editores.

Franco, S y otros. (2000). La práctica pedagógica de los maestros inmersa en los escenarios de gestión curricular. Medellín: Producciones Infinito.

Gruppe, Ommo, (1976). Teoría pedagógica de la Educación Física. Madrid: INEF.

Haag, Herbert. (1995). Fundaciones teóricas de la ciencia del Deporte como disciplina científica. Contribución para una filosofía (meta-teoría) de una ciencia del Deporte. Verlag Karl Hofmann, Schorndorf (Serie ICSSPE Sport Science Studies n. ${ }^{\circ}$ 6), 167 pp. 1994. Traducción de Maurer Furst Antonio. Valparaíso, Chile.

Max Neef, M. A. y otros. (1997). Desarrollo a Escala Humana. Una opción para el futuro. Medellín: Proyecto 20 Editores.

Molina, V. y otros. (2001). Didáctica contemporánea, Motricidad comunitaria y Ocio. Medellín: Editorial Universidad Pontificia Bolivariana.

\footnotetext{
6 Para la realización de esta tarea será de gran ayuda la conformación del grupo de investigación denominado Historia y Epistemología de la Educación Física, la Recreación y el Deporte
} 
Morales R., Leonel. (1990, enero-diciembre). Elementos para una aproximación al trabajo teórico de la Educación Física Colombiana. En Revista Educación Física y Deporte,12(1-2). Medellín: Universidad de Antioquia, Instituto Universitario de Educación Física y Deporte.

Moreno, William, G. y otros. (1995, mayo ). Grupo de trabajo académico GUTAC. Microcurrículo en Pedagogía Motriz. Convenio SEDUCA, Medellín: Universidad de Antioquia.

Nussbaum, M. y Sen, A. (1993). La calidad de vida. México: The United Nations University-Fondo de la Cultura Económica.

Parlebas, Pierre. (1992). Educación Física Moderna y Ciencia de la Acción Motriz. En Congreso Panamericano de Educación Física (XIII: 1992: Bogotá). Ponencia del XIII Congreso Panamericano de Educación Física. Educación Física Moderna y Ciencia de la Acción Motriz. Universidad Pedagógica Nacional. Bogotá.

Pinillos, G. Jesús María y otros. (2005). Diseño participado de guías curriculares para maestros(as) en el área de la Educación Física, la Recreación y el Deporte en la educación básica y media en el Departamento de Antioquia, teniendo como referente un modelo pedagógico y las nuevas tecnologías de la información y la comunicación. Investigación. Medellín: Universidad de Antioquia.

Pinillos, G. Jesús María. (1999). Pedagogía y Didáctica de la Educación Física. En Congreso Panamericano de Educación Física. (XII: 1999. Panamá, Panamá). Ponencia del XII Congreso Panamericano de Educación Física.

Pinillos G., Jesús María. (2004). La función del educador físico en la institución escolar. En Primer Seminario Taller Pedagogía, Metodología y Didáctica de la Educación Física en la Educación Básica Primaria (1: 2004. Rionegro, Antioquia) Ponencia del Primer Seminario Taller Pedagogía, Metodología y Didáctica de la Educación Física en la Educación básica primaria. Rionegro, Antioquia: Politécnico Colombiano Jaime Isaza Cadavid.

Pinillos G., Jesús María. (2002, diciembre). La evaluación por competencias en el área de la Educación Física, la Recreación y el Deporte. En Revista Kinesis, 35.

Pinillos G. Jesús María. (2002, 31 de octubre y 1de noviembre). Un currículo basado en la pedagogía problémica. Ponencia. En Seminario Taller Sobre Currículo". Bogotá: Universidad Pedagógica Nacional, Facultad de Educación Física.

Pinillos G., Jesús María. (2004). La Educación Física y el Deporte una oposición de discursos en Colombia, en el periodo comprendido entre 1968 y 1991.Trabajo de grado Maestría. Medellín: Universidad de Antioquia, Facultad de Educación.

Quesada de la Cruz. Patricia y Sánchez C. Néstor Alonso. (1991). La Educación Física una Historia por construir. Trabajo de grado pregrado. Cali: Universidad del Valle.

Ramírez, Humberto y otros. (1997). El niño sano. Medellín: Universidad de Antioquia.

Ramos, Santiago. (1989, julio). El ejercicio profesional del Licenciado en Educación Física en el campo del Entrenamiento Deportivo. En Congreso Colombiano de Educación Física. (III: 1988. Cartagena). Ponencia presentada al III Congreso Colombiano de Educación Física. En Revista Colombiana de Educación Física, 4. Bogotá: Asociación Colombiana de profesores de Educación Física.

Rodríguez López, Juan. (1997). Deporte y Ciencia: teoría de la actividad física. Madrid: INDE.

Rodríguez, Hilda Mar. (2002). Encuentro con la pedagogía. De lo clásico a las tendencias de hoy. En Parcelas y horizontes un en cuentro con las disciplinas. Medellín: Grupo BioantropologíaReproducción-Biogénesis. Universidad de Antioquia.

Roldan, Ofelia. (1997). Educación, Desarrollo Humano y Cultura Física. Documento.

Ruiz, Luís M. (1995). Competencia motriz. Madrid: Gimnos.

Sáenz, Pedro y López Buñuel. (1997). La Educación Física y su didáctica. Sevilla: Wanculen.

Sergio, Manuel. (1987). Para una Epistemología de la Motricidad Hu mana. Lisboa: Compendiun.

Tabares, F., José Fernando. (2006) El Ocio como dispositivo para la potenciación del desarrollo humano. Corporación civitas.Funlibre. Centro de documentación virtual en Recreación, Tiempo Libre y Ocio. En: http://www.funlibre.org/documentos/conceptuales.html. Consultado el 28 de agosto. Centro de Documentación Virtual en Recreación, Tiempo Libre y Ocio

Universidad Pedagógica Nacional, Departamento de Educación Física. Comité de Currículo. (1985). Plan de Estudios de la Licenciatura en Educación Física. Bogotá.

Uribe Pareja, Iván Darío y otros. (2004). La Pedagogía de la motricidad como estrategia para la promoción de la salud. Trabajo de grado maestría. Medellín: Universidad de Antioquia, Facultad de Enfermería.

Uribe Pareja, Iván Darío y otros. (2004). Sentidos de la motricidad en la promoción de la salud. Percepciones desde las familias en los municipios de Alejandría y Guatape. Medellín: Soluciones Editoriales.

Velásquez, Luz Estela y otros. (2004). Aprovechamiento creativo del tiempo libre como estrategia pedagógica en la formación del maestro culto. Núcleo pedagogía del cuerpo. Envigado: Institución educativa Normal Superior de Envigado.

Velásquez, María Teresa. (2000). Psicomotricidad. Bogotá. Cátedra UPN. 1974. Retomada por Ministerio de Educación Nacional. Lineamientos Curriculares Educación Física, Recreación y Deportes. Bogotá: Cooperativa Editorial Magisterio.

Weinstein, L. (1978). Salud y autogestión. Montevideo: Nordan y Buenos Aires, Tupac Ediciones.

Weistein, L. El desarrollo de la salud y la salud del desarrollo. Nordan: Montevideo.

Zuluaga Garcés, O. L. (1987). Pedagogía e historia. Bogotá: Foro Nacional por Colombia. 